\title{
The Impact of RFID Investment on Complex Product in Three-Level Assembly Supply Chain
}

\author{
Wei Xu, ${ }^{1}$ Zhaotong Lian, ${ }^{1}$ and Xifan Yao ${ }^{2}$ \\ ${ }^{1}$ Faculty of Business Administration, University of Macau, Macau \\ ${ }^{2}$ School of Mechanical and Automotive Engineering, South China University of Technology, Guangzhou, China \\ Correspondence should be addressed to Zhaotong Lian; lianzt@umac.mo
}

Received 15 March 2013; Accepted 20 May 2013

Academic Editor: Qingsong Xu

Copyright (c) 2013 Wei Xu et al. This is an open access article distributed under the Creative Commons Attribution License, which permits unrestricted use, distribution, and reproduction in any medium, provided the original work is properly cited.

\begin{abstract}
Motivated by the complex product with the feature about error-prone assembly system and supply chain inventory inaccuracy, this paper elaborates on the impact of information technology investment on complex product by establishing a three-stage supply chain model involving two suppliers, one manufacturer, and retailer which carried out Stackelberg games. In addition, it not only compares the manufacturer and the retailer's optimal decision and maximum profit under the situation of the information asymmetry and free information sharing, but also analyzes their market behavior and changes in market performance. Meanwhile, it points out that the downstream in supply chain masters more information about market demands compared to the upstream one. The optimal cost threshold values of technology investment are also examined both for the centralized and the decentralized scenarios utilizing quantitative and modeling methods. By analyzing and comparing the optimal profit with or without investment on information technology, it establishes a supply chain coordination model which boosts the application of information technology. At the same time, it offers the conditions on which the upstream and downstream enterprises can coordinate with one another. The results of this paper have contributed significantly to making the price and ordering decisions on whether RFID should be adopted among members of the supply chain. Finally, we present numerical analyses, and several extensions of the model are considered as well.
\end{abstract}

\section{Introduction}

Represented typically by autoindustry, complex product assembly process is characterized by typical discrete manufacturing. It involves numerous components and parts, miscellaneous technology, and high-accuracy requirement. The assembly system has the characteristics of mixed production. However, these complex product assembly operations are mainly completed manually with error-prone steps. Due to the limits of technical investment, the range of assembly monitoring is only restricted to the executive process itself, and it neglects logical relationship between the assembly task and logistics task so that the assembly and logistics cannot be managed uniformly, thus causing the disconnection between material flow and information flow. Since it is easy to get wrong and timeconsuming, the information technology is required to ensure that the architectural decisions will be implemented correctly.

To solve the problems above, the model of assembly system involving the assembly agent nodes and logistics agent nodes must be established so as to realize uniform monitoring and management. Additionally, RFID can identify items conveniently by virtue of the characteristics of noncontact, remote distance and read-write so as to improve the real time of assembly monitoring and promote synchronization of material flow and information flow. What is more, the changes in assembly states and logistics states can be reflected through generation, destruction, and update of RFID tag. In that case, if assembly agent and logistics agent can be modeled uniformly to establish the mapping relation between RFID and assembly executive state, the feasibility of assembly executive process can be enhanced. 
Obviously, the wide application of information technology will free people from the heavy labour. Some largescale retail enterprises, such as Wal-Mart and METRO, have obtained obvious effects by trying out RFID technology in the aspects of clothes and daily necessities. However, the high cost of RFID equipment limits its wider popularization and application to more supply chains and enterprises. Therefore, how to coordinate the upstream and downstream firms in the assembly system to use the RFID technology to improve the performance of the supply chain is of great significance.

According to the report of American Shipper, the average occurrence rate of demand exceeding supply and out-ofstock situations is $8 \%$ in global retail business. Surprisingly, for a $8000 \mathrm{~m}^{2}$ retail store with the annual sales volume of RMB 150 million, it will lose RMB 8.4 million sales volume only due to the stockout per year. The replenishment speed is 3 times faster than that of goods without the tags. After Wal-Mart adopts electronic product code (EPC) supported by RFID technology to monitor goods, the stockout rate reduces $16 \%$. For the goods tagged with RFID labels, manual ordering reduces by $10 \%$. RFID can decrease the quantity of safety inventory effectively and make quick response to the stockout [1]. After BestBuy adopts RFID, goods availability has been added to $93 \%$ from $80 \%$. At present, a famous British retailer TESCO introduces a device called smart shelf which can record each move of all products on the shelves. This is also one of applications of RFID technology. At the same time, RFID antennae are installed at the entrance and exit of the warehouse, which are connected with warehouse management system. Thus, when the goods enter and leave the warehouse, electronic label information can be read promptly. Meanwhile, the most accurate goods data can be gained easily. Additionally, the accurate information about the inventory quantity can be obtained as well. The study of American organs concerned shows that the accuracy rate of the inventory can reach $99.5 \%$ for the retail enterprises applying information technology to inventory management, while other enterprises can only reach $80 \%-85 \%$ $[2,3]$.

With the help of information technology, enterprise informatization can be improved; meanwhile, industrial information sharing can be promoted. Real-time dynamic indicators in the supply chain, such as inventory analysis, market and customer analysis, and other data need monitoring by RFID sensor and are transmitted to the server through internet network. Thus, data information from different channels is gathered through cloud calculating data center of the internet of things so as to draw information strategies igniting the profit point.

Although complex product can be traced more accurately in real time with RFID technology, one of the major obstacles to adoption is the cost of RFID implementation. RFID technology incurs huge cost including not only the installation investment but the costs of tags and readers as well. Motivated by the RFID investment issue, we aim to explore the prospects by mainly focusing on the optimal investment strategies in order to maximize the profits under conditions with or without the perishable products value tracing when the demand is stochastic in a two-level supply chain involving a manufacturer and a retailer. We consider both the centralized and decentralized scenario.

Particularly, the main research questions that this paper addresses are as follows.

(i) What are the levels of the optimal investment that make RFID adoption economically feasible in threelevel centralized and decentralized assembly supply chains?

(ii) How to combine the actual supply chain problem, such as the shelf shrinkage, errors in assembly process, and misplaced products, to implement the technical innovation investment to be able to realize greater investment returns?

The remainder of this paper is organized as follows. In the following section, we give a quick idea of the topics covered in the literature review. In addition, literatures related to this study will be reviewed. In Section 3, we introduce the system architecture and benefit evaluation, mainly involving assembly manufacturing system based on multi-agent and RFID. The model analysis and the main results of this research are presented in detail in Sections 4 to 6 . The numerical analysis is examined in Section 7, and we offer a summary, together with a critique of the model, and a direction for future research in the final section.

\section{Literature Review}

RFID is a term that has been around for several years. In recent years, the analysis of RFID technology investment on various industries has attracted the attention of more and more scholars. As an exploratory research in essence, more specially, the investment analysis based on mathematical models is extremely scarce. This section mainly builds on three streams of literature: the potential benefits and most common application of RFID technology, RFID investment on supply chain with contracts, and two- or three-level supply chain coordination, as well as the development of an RFID and multi-agent system based on manufacturing control for industrial applications and dynamic logistics process.

The transaction and transportation errors, misplacement, delay delivery, and shrinkage errors are the main causes of inventory inaccuracy, all of which have been discussed as areas where RFID can increase accuracy. There are numerous papers in the literature that have considered the advantages of RFID technology. The benefits under different categories are presented as follows. Lee and Özer [4] study a singleitem, periodic-review inventory system with replenishment policy $(s, S)$. They integrate RFID technology with uncertain demands and random distribution of transaction errors and observe the inventory cost related to transaction errors. Atali et al. [5] extend the abovementioned models, they develop two models based on shrinkage such as thefts and damages, misplacement, and transaction or scanning errors. They consider that RFID can effectively reduce 


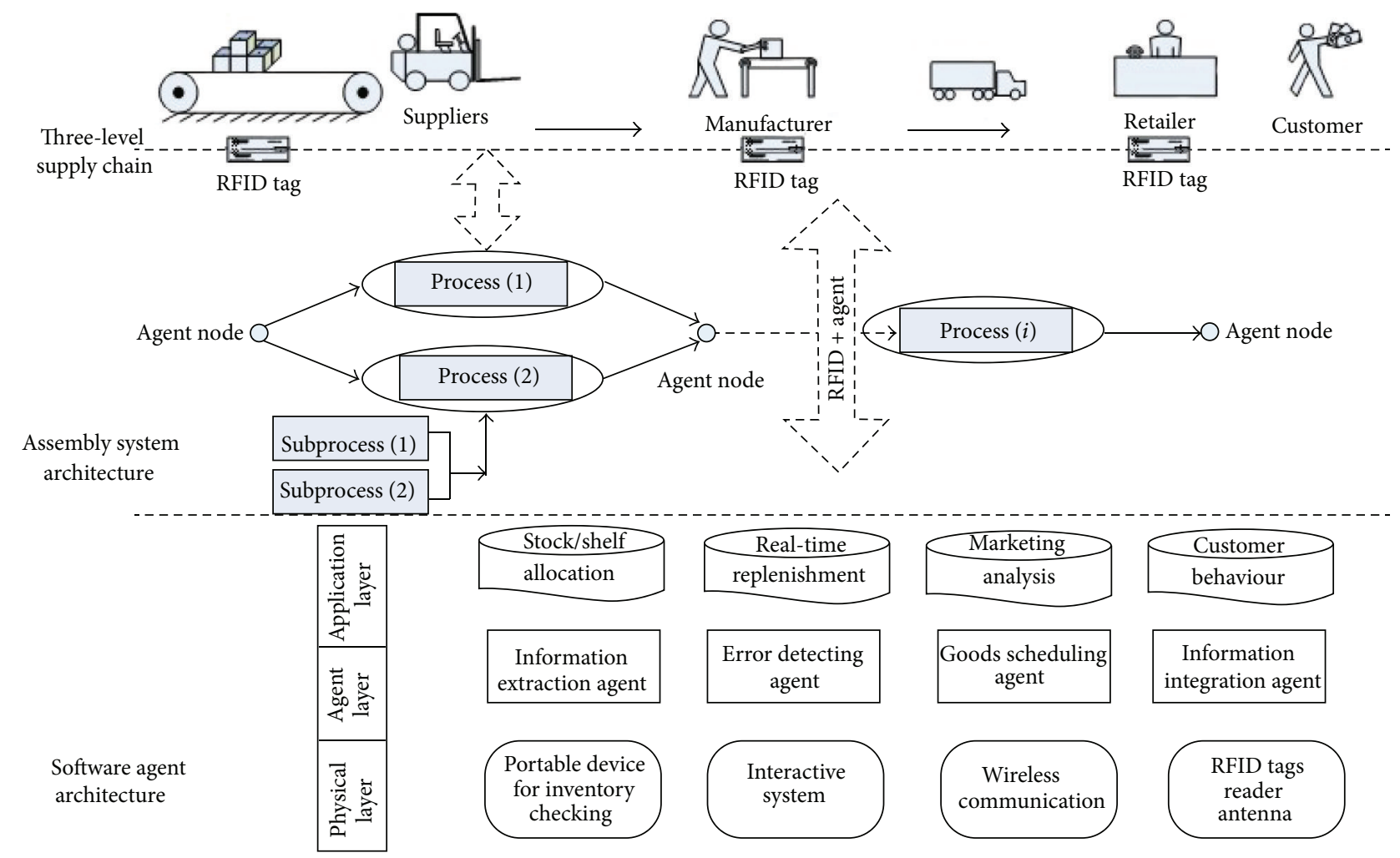

FIGURE 1: Architecture of the information technology in complex product assembly system.

the mistake rate and improve inventory visibility. Tajima [6] first mentions that the shrinkage errors include employee theft, shoplifting, administration and paperwork errors, vendor fraud, and unavailable products for sale. Besides, RFID has been discussed as a solution to black market sales, which could also improve inventory records by reducing human errors in material handling (as discussed elsewhere $[7,8])$. Ngai et al. [9] analyze a case study on mobile commerce system based on RFID technology with strong function on locating, tracking, and managing the containers. Gaukler et al. [10] focus on the investment cost of RFID technology and assume that the RFID tag cost can be shared by all supply chain members. Besides, they argue that RFID can improve stock control policies as well as inventory replenishment policies. They extend the previous model to analyze an item-level RFID application in twolevel supply chain. They propose two cases of centralized and decentralized decision with or without RFID and mainly discuss how the manufacturer and the retailer can optimize their own profits without cooperation. Heese [11] indicate that RFID technology is more beneficial in decentralized supply chains. Although RFID cannot eliminate all errors, it can be quickly detected and effectively handled. Several authors, such as Dutta et al. [12], Rekik et al. [13], Szmerekovsky and Zhang [14], Bottani and Rizzi [15], Tu et al. [16], Wang et al. [17], and Whitaker et al. [18], were attracted by its use of eliminating these errors. They all pay close attention to the impact of RFID on inventory inaccuracy due to variety of errors.

\section{Overview of System Architecture and Benefit Evaluation}

In this section, we mainly discuss the system platform architecture based on multi-agent and RFID. Combining these two new technologies together, it has become one of the potential candidates for all assembly manufacturing and supply chain systems in the future.

As is shown in Figure 1, all the assembly transition and logistics distribution are guided and controlled, respectively, through multi-agent, and it is like the human brain, which not only improves agility of manufacturing system, but also makes manufacturing system characterized by heterogeneous and geographically distributed. In addition, in order to improve the efficiency of raw material identification, the material can be identified with RFID tag. When the parts set needed by some assemblies are taken out from the warehouse or some assembly tasks are completed, RFID tags are immediately adopted to identify the parts set or new assembly. When the materials are transported to the destination according to the design route, the content of tag should be updated. The construction and update of RFID tag are unified with events in assembly executive process. 


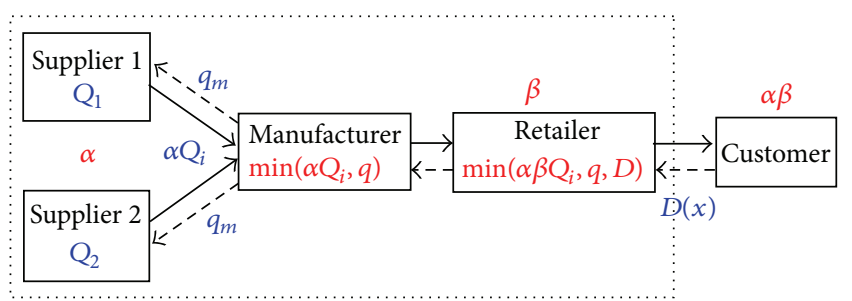

Figure 2: The schematic diagram of system model.

The assembly executive state can be reflected through tag state. In that case, all transitions can be regarded as mutuallycoordinated and mutuallyindependent equal entity through certain protocol with certain limits of authority. Besides, each entity has certain structural patterns and completes different work driven by respective local data.

The control system may be realized through a multiagent system mainly including assembly agent and logistics agent. Figure 1 shows functional models of the two types of agent. It can be seen that assembly agent firstly reads RFID tag information, gives assembly task promptly, then reads task scheduling and assembly technology, respectively, from assembly database, and conducts guide and process inspection control of assembly operation. After the assembly is finished, task information in the database should be updated. Then, the logistics agent identifies RFID and obtains material information from assembly database and then guides the transport process. By using distributed database to realize distributed ontology, we can ensure the distributivity of the system, the real-time updating, and data consistency of the system. As mentioned before, the automotive assembly management is based on multi-agent and RFID, which includes application layer, agent layer, and physical lay. The bottom module is mainly responsible for the processing of various businesses in the whole system and the transmission of various data into upper-layer management system after saving them in the database; besides, the data collection and processing mainly aim to collect various data in field production. The intermediate layer is responsible for monitoring the adaptive configuration of field collection nodes (intelligent terminal) to realize the collection and filtration of RFID data, to monitor collection event of primary layer and information event of upper-layer system and to command parsing and sending the data into business logic layer.

\section{Notations and Model Scenarios}

This paper targets the assembly system and supply chain involving two suppliers, one manufacturer and one retailer. The schematic diagram of system model is shown as illustrated Figure 2. The theoretical framework of assembly supply chain is established on a hypothesis that information is symmetric and complete; we also assume the partners are completely rational and the market is completely competitive. The following summary of notations shown at the end of the paper are used in this paper.
In order to explore the description and notation of this system, there are two different scenarios for our model: the centralized scenario and the decentralized one. Under the first one, it is assumed that a central decision maker determines whether or not to invest in information technology and the order quantity to maximize the total profit. In the other scenario, there is no cooperation or information sharing among the suppliers, the manufacturer and the retailer. Each member tries to pursue his own maximum profits.

Under the above-discussed structure, there are two subsituations: whether the information technology station is in the assembly supply chain or not. Once the information system is adopted, it will contribute to optimizing the goods stored in warehouse and ensure inventory accuracy and safety.

Therefore, we can consider using real-time monitoring technology which is based on multi-agent and RFID to help enterprises monitor inventory accurately and avoid losses of enterprises. However, within the shrinkage of the error-prone assembly system and supply chain inventory inaccuracy, the cost from technology investment has risen up dramatically. In this paper, we only consider the variable cost resulted from the application of the multi-agent and RFID technology, that is, the cost of multi-agent public software platform and RFID $\operatorname{tag} c_{t}$. When the retailer employs the technology, that is $\beta=1$, the complex production assembly system of upstream suppliers and the manufacturer will not be improved by these so that the maximum effect of the technology in the supply chain could not be exerted. When the suppliers adopt multi-agent and RFID, that is, $\alpha=1$, the downstream retailers can use the RFID labels tagged on commodities by the manufacturer, which is beneficial to the retailer from this $(\beta=1)$. At this time, the suppliers or the manufacturer undertake the technology investment alone; however, the retailer can free-ride all these. Thus the supplier's enthusiasm for implementing multi-agent and RFID is totally weakened. To make the upstream have a growing interest in investing in information technology, reduce the losses resulted from inaccurate inventory, and increase the performance of the supply chain, the downstream in supply chain can encourage the suppliers and the manufacturer with some compensations and reduce the impacts of the free-ride effect. Based on this, this paper will also study the supply chain coordination when multi-agent and RFID technology are invested among the three members.

\section{Optimal Strategy in Centralized Scenario}

In order to achieve the highest performance of the whole assembly supply chain, all the members make their own decision together. Therefore, it is necessary to analyze the conditions on which the optimal technology investment is implemented in centralized scenario.

\subsection{Optimal Decision in Centralized Supply Chain without Multi-Agent and RFID Technology}

5.1.1. Two Suppliers with the Same Yields. When the order quantity is $Q$ and the actual inventory which can be used to 


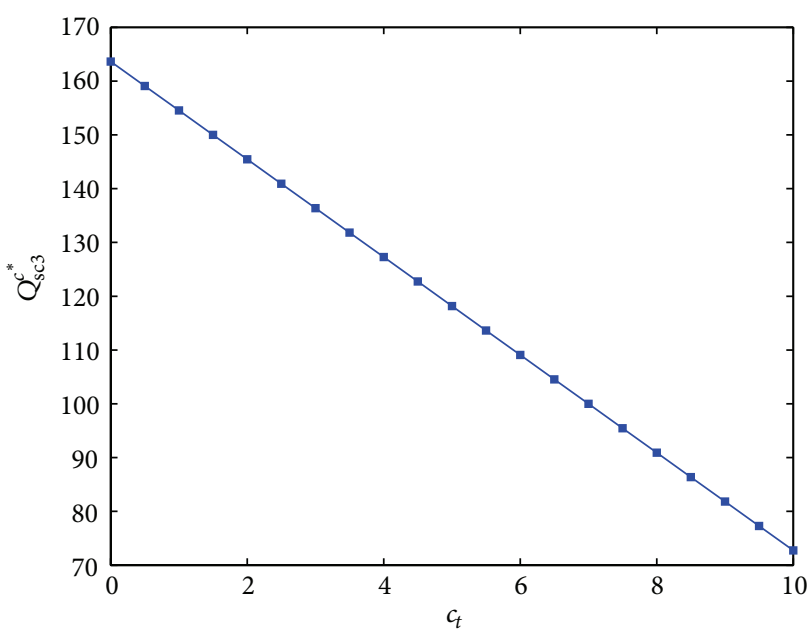

(a)

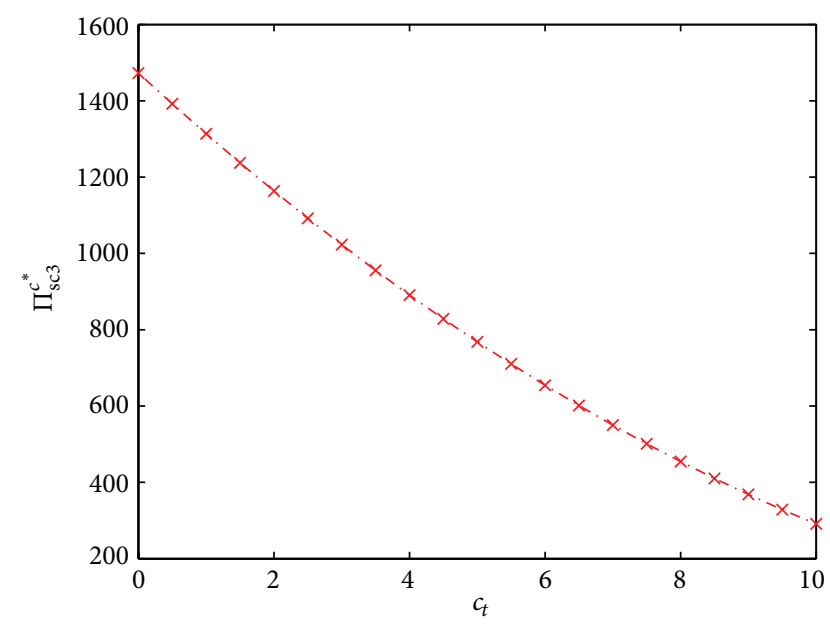

(b)

FIGURE 3: Variation of $Q_{\mathrm{sc3}}^{c^{*}}$ and $\Pi_{\mathrm{sc3}}^{c^{*}}$ with $c_{t}$.

satisfy consumers' demands by the normal sales links is $\alpha \beta Q$, the expected sales of whole supply chain are $E \min (D, \alpha \beta Q)$; meanwhile, the surplus is $E(\alpha \beta Q-D)^{+}$. Then we can easily get the expected profit of the whole supply chain:

$$
\Pi_{\mathrm{scl}}^{c}=p_{m} \min (D, \alpha \beta Q)+v[\alpha \beta Q-D]^{+}-2 c Q .
$$

Using the following equalities:

$$
\begin{aligned}
E \min (D, \alpha \beta Q)= & \alpha \beta Q-\int_{0}^{\alpha \beta Q}(\alpha \beta Q-x) f(x) d x, \\
E(\alpha \beta Q-D)^{+} & =\alpha \beta Q-E \min (x, \alpha \beta Q) \\
& =\int_{0}^{\alpha \beta Q}(\alpha \beta Q-x) f(x) d x,
\end{aligned}
$$

the expected profit function associated with the same yields of two suppliers is given by

$$
\begin{aligned}
\Pi_{\mathrm{scl}}^{c}=p_{m} \int_{\underline{\alpha}}^{\bar{\alpha}} \int_{\underline{\beta}}^{\bar{\beta}}\left[\alpha \beta Q-\int_{0}^{\alpha \beta Q}(\alpha \beta Q-x) f(x) d x\right] \\
\times g(\beta) h(\alpha) d \beta d \alpha \\
+s \int_{\underline{\alpha}}^{\bar{\alpha}} \int_{\underline{\beta}}^{\bar{\beta}}\left[\int_{0}^{\alpha \beta Q}(\alpha \beta Q-x) f(x) d x\right] \\
\times g(\beta) h(\alpha) d \beta d \alpha-2 c Q .
\end{aligned}
$$

Such an assumption which is explored by several authors, such as Heese [11], enables us to assume that the customer demand is subject to uniformly distribution. Therefore, in fact, the above equation can be simplified further to

$$
\begin{aligned}
\Pi_{\mathrm{scl}}^{c}= & p_{m} \mu_{\alpha} \mu_{\beta} Q-\frac{p_{m}-s_{m}}{4 \mu_{x}} \cdot\left(\sigma_{\alpha}^{2}+\mu_{\alpha}^{2}\right) \\
& \times\left(\sigma_{\beta}^{2}+\mu_{\beta}^{2}\right) Q^{2}-2 c Q .
\end{aligned}
$$

Next, we calculate the second derivative about $Q$ and know that $\partial^{2} \Pi_{s c 1}^{c} / \partial Q^{2}<0$; therefore, $\Pi_{s c 1}^{c}$ is a concave function about $Q$. In the particular case of a uniformly distributed demand, the optimal order quantity and optimal expected profit of the whole supply chain can be written as follows:

$$
\begin{aligned}
Q_{\mathrm{scl}}^{c *} & =\frac{2 \mu_{x}\left(p_{m} \mu_{\alpha} \mu_{\beta}-c\right)}{\left(p_{m}-s_{m}\right)\left(\sigma_{\alpha}^{2}+\mu_{\alpha}^{2}\right)\left(\sigma_{\beta}^{2}+\mu_{\beta}^{2}\right)}, \\
\Pi_{\mathrm{scl}}^{c *} & =\frac{\left(p_{m} \mu_{\alpha} \mu_{\beta}-c\right)^{2} \mu_{x}}{\left(p_{m}-s_{m}\right)\left(\sigma_{\alpha}^{2}+\mu_{\alpha}^{2}\right)\left(\sigma_{\beta}^{2}+\mu_{\beta}^{2}\right)} .
\end{aligned}
$$

The Figures 3(a) and 3(b) represent the variation of $Q_{s c 3}^{c *}$ and $\Pi_{\mathrm{sc3}}^{c *}$ with RIFD investment cost $c_{t}$ respectively.

5.1.2. Two Suppliers with Different Yields. There are some problems within the current operational process which make the internal production not well synchronized, so the two suppliers with different yields are also discussed as follows

$$
\begin{aligned}
\Pi_{\mathrm{sc} 2}^{c}= & p_{m} \cdot \min \left(\alpha \beta Q_{1}, \alpha \beta Q_{2}, D\right) \\
& -s_{m}\left[D-\min \left(\alpha \beta Q_{1}, \alpha \beta Q_{2}\right)\right]^{+} \\
& +v\left[\min \left(\alpha \beta Q_{1}, \alpha \beta Q_{2}\right)-D\right]^{+} \\
& -c\left(Q_{1}+Q_{2}\right) .
\end{aligned}
$$


For the different yield problem of two suppliers, in order to derive the optimal order decision, we must distinguish the different positions of the distributions of $D(x)$ and $\alpha \beta Q_{i}$ to get the expression of the expected profit. Then, the above equation for each configuration is shown in

$$
\Pi_{\mathrm{sc2}}^{c}= \begin{cases}\Pi_{\mathrm{sc2}}^{c}=p_{m} \alpha \beta Q_{1}-s_{m}\left(D-\alpha \beta Q_{1}\right)-c\left(Q_{1}+Q_{2}\right), & \alpha \beta Q_{1}<\alpha \beta Q_{2}<D \text { or } \alpha \beta Q_{1}<D<\alpha \beta Q_{2}, \\ \Pi_{s c 2}^{c}=p_{m} \alpha \beta Q_{2}-s_{m}\left(D-\alpha \beta Q_{2}\right)-c\left(Q_{1}+Q_{2}\right), & \alpha \beta Q_{2}<\alpha \beta Q_{1}<D \text { or } \alpha \beta Q_{2}<D<\alpha \beta Q_{1}, \\ \Pi_{s c 2}^{c}=p_{m} D+v\left(\alpha \beta Q_{2}-D\right)-c\left(Q_{1}+Q_{2}\right), & D<\alpha \beta Q_{2}<\alpha \beta Q_{1}, \\ \Pi_{s c 2}^{c}=p_{m} D+v\left(\alpha \beta Q_{1}-D\right)-c\left(Q_{1}+Q_{2}\right), & D<\alpha \beta Q_{1}<\alpha \beta Q_{2} .\end{cases}
$$

To refresh the equation set, we obtain the optimal expected profit of the whole supply chain:

$$
\begin{aligned}
\Pi_{\mathrm{sc} 2}^{c *}= & \left(p_{m}+s_{m}-v\right) \cdot \min \left(\alpha \beta Q_{1}, \alpha \beta Q_{2}, D\right) \\
& -s_{m} D+v \cdot \min \left(\alpha \beta Q_{1}, \alpha \beta Q_{2}\right)-c\left(Q_{1}+Q_{2}\right)
\end{aligned}
$$

5.2. Optimal Decision in Centralized Supply Chain with MultiAgent and RFID Technology. Next, we investigate the centralized system with technology input in detail. The RFID system can identify items conveniently by virtue of the characteristics of noncontact, remote distance, and read-write so as to improve the real time of assembly field monitoring and promote synchronization of logistic flow and information flow. What is more, multi-agent system including agent assembly nodes and agent logistics nodes can be modeled uniformly to establish the mapping relation between RFID tag and assembly executive state. Moreover, the feasibility of assembly executive process can be enhanced so as to get real time and accuracy of assembly manufacturer process monitoring. Under such circumstances, all synchronization progress, status, and any errors in assembly supply chain process can be reflected through the updated information of RFID tag.

Above all, as multi-agent and RFID system provides an accurate information of the current inventory, it contributes to the monitoring and management of the products in realtime. Meanwhile, there is also the technology investment cost $c_{t}$. Therefore, the unit purchasing cost is $c+c_{t}$. The excepted profits are as follows

$$
\Pi_{\mathrm{sc} 3}^{c}=p_{m} \min (D, Q)+s_{m}[Q-D]^{+}-2\left(c+c_{t}\right) Q
$$

where $E \min (x, Q)=Q-\int_{0}^{Q}(Q-x) f(x) d x$ and $[x-Q]^{+}=$ $Q-E \min (x, Q)=\int_{0}^{Q}(Q-x) f(x) d x$. Since $\partial^{2} \Pi_{s c 3}^{c} / \partial Q^{2}=$ $-p_{m}-s_{m} / 2 \mu_{x}<0$, the preceding equation is a concave function about $Q$ that is, it has the only optimal solution $Q^{*}$, which achieves the maximum profit of the whole supply chain.
Differentiating $\Pi_{\mathrm{sc3}}^{c}$ with respect to $Q$, the optimal ordering quantity and profit of the whole supply chain are given by

$$
\begin{aligned}
Q_{\mathrm{sc} 3}^{c *} & =2 \mu_{x} \frac{p_{m}-c-c_{t}}{p_{m}-s_{m}}, \\
\Pi_{\mathrm{sc} 3}^{c *} & =\frac{\left(p_{m}-c-t\right)^{2}}{p_{m}-s_{m}} \mu_{x} .
\end{aligned}
$$

5.3. The Advantages of Deployment with Technology Investment. In this section, we focus on seeking answers from the following questions: how to solve such issues as the assembly manufacture errors and shelf shrinkage by means of multi-agent and RFID system more effectively? What are the levels of optimal investment that enjoy the technology economically feasible in centralized supply chain?

Since all members take the overall interests of the whole supply chain into account, they pursue the global profit maximization and carry on the technology innovation with information sharing in the centralized scenario. As we all know, if they do not introduce technologies, they will suffer the impacts of assembly errors and inaccurate inventory. Conversely, they implement it but undertake the technical cost.

To analyze the profit and loss with technology investments, the benefit function $\Delta \Pi^{c}$ is introduced. Clearly, when $\Delta \Pi^{c}=0$, there exists a break-even point $c_{t}^{\prime}$ which makes technical investment always profitable. In other words, the increased profits generated by innovative technology are always larger than the investment cost of multi-agent software and RFID tags. In detail, one essential condition is to satisfy the inequality $\Delta \Pi^{c}>0$.

Proposition 1. The threshold value of technology investment cost is $c_{t}^{\prime}=p_{m}-c-\left(\left(p_{m} \mu_{\beta} \mu_{\alpha}-c\right) / \sqrt{\left(\sigma_{\beta}^{2}+\mu_{\beta}^{2}\right)\left(\sigma_{\alpha}^{2}+\mu_{\alpha}^{2}\right)}\right)$. When $c_{t}<c_{t}^{\prime}$, the optimal solution is to make investment for situation in the centralized scenario, when $c_{t}>c_{t}^{\prime}$, without any investment on innovative technology being beneficial for the whole supply chain.

As can be seen from the above expression, we find that $c_{t}$ has relationship not only with $\mu_{\alpha}$ and $\mu_{\beta}$, but also with the selling price of manufacturer as well. It further proves that the higher the selling price $p_{m}$ is, the higher the technology 


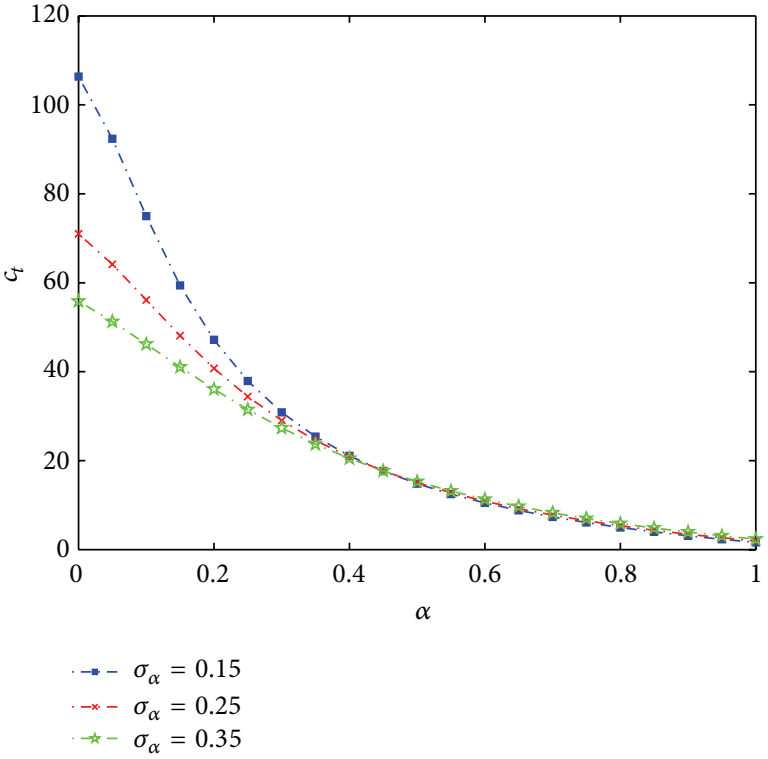

FIGURE 4: Variation of $c_{t}$ with $\alpha$ for different values of $\sigma_{\alpha}$.

cost that can be accepted by the supply chain system will be. Meanwhile, for a given $\mu_{\alpha}, \Delta \Pi$ can be greater or less than 0 depending on the value of $\mu_{x}$. It appears that the benefit achieved by the deployment of the multi-agent and RFID system is comparable to the profit without any technology investment. Figure 4 from the analysis above represents the variation of $c_{t}$ with $\alpha$ for different values of $\sigma_{\alpha}$. The more the customer demand and the less effective the assembly system is (that is the error rate $\alpha$ is higher), the more important the technology investment will be for the decision whether to adopt the information technology, and the members in assembly supply chain can be benefited from the ordering quantity if we implement it. Additionally, the comparison of the Figure 5 reveals the variation of $\Delta Q$ and $\Delta \Pi$ with $\alpha$ for different demand.

\section{Optimal Strategy in Decentralized Scenario}

In this section, we mainly evaluate and analyze the impact of technology investment among three supply chain partners. Owning to independence relation, they pursue the maximum profits, respectively. Consequently, the main issue will be focused on which partner takes the priority to employ multi-agent and RFID system and bear the higher costs. Then two approaches are presented. Similar to the previous section, the optimal benefits with or without adoption of technology by numerical solutions under the decentralization are also proposed. In addition, the retailer places an order decision in terms of the wholesale price charged by the manufacturer which book from the supplier, and the optimal order quantity has been the focus of description and explanation.

\subsection{Optimal Decision in Decentralized Supply Chain without Multi-Agent and RFID Technology}

6.1.1. The Basic Model of Two Suppliers. According to the above analysis, the profit function of supplier $i$ that normally depended on $Q_{i}$ in decentralized scenario is given by

$$
\Pi_{s_{i}}^{D}=w_{i} \cdot \min \left(\alpha Q_{i}, q\right)-s_{i}\left[q-\alpha Q_{i}\right]^{+}-\sum_{i=1}^{2} c Q_{i} .
$$

To make analysis and computation more convenient, the holding cost incurred by the supplier $i$ with less actual quantity delivered can be omitted. Then we assume $k_{i}(x) \equiv$ $\int_{0}^{x} \alpha_{i} d H_{i}\left(\alpha_{i}\right)$, according to the following formulas deduced:

$$
\begin{aligned}
E\left[\min \left(\alpha_{i} Q_{i}, q\right)\right]= & \int_{0}^{q / Q_{i}} \alpha_{i} Q_{i} h_{i}\left(\alpha_{i}\right) d \alpha_{i} \\
& +\int_{q / Q_{i}}^{1} q h_{i}\left(\alpha_{i}\right) d \alpha_{i} \\
= & Q_{i} k_{i}\left(\frac{q}{Q_{i}}\right)+q H_{i}\left(\frac{q}{Q_{i}}\right), \\
E\left[\left(q-\alpha_{i} Q_{i}\right)^{+}\right]= & \int_{0}^{q / Q_{i}}\left(q-\alpha_{i} Q_{i}\right) h_{i}\left(\alpha_{i}\right) d \alpha_{i} \\
= & q H_{i}\left(\frac{q}{Q_{i}}\right)-Q_{i} k_{i}\left(\frac{q}{Q_{i}}\right) .
\end{aligned}
$$

We can easily get the expected profit of supplier:

$$
\begin{aligned}
E\left[\Pi_{s_{i}}^{D}\right]= & w_{i}\left[Q_{i} k_{i}\left(\frac{q}{Q_{i}}\right)+q H_{i}\left(\frac{q}{Q_{i}}\right)\right] \\
& -s_{i}\left[q H_{i}\left(\frac{q}{Q_{i}}\right)-Q_{i} k_{i}\left(\frac{q}{Q_{i}}\right)\right] \\
& -\sum_{i=1}^{2} c Q_{i} .
\end{aligned}
$$

The first partial derivative of $\Pi_{s_{i}}^{D}$ with respect to $Q_{i}$ can be written as

$$
\begin{aligned}
\frac{\partial \Pi_{s_{i}}^{D}}{\partial Q_{i}}=w_{i}[ & k_{i}\left(\frac{q}{Q_{i}}\right)+Q_{i} \frac{q}{Q_{i}} h_{i}\left(\frac{q}{Q_{i}}\right)\left(-\frac{q}{Q_{i}^{2}}\right) \\
& \left.-Q_{i} \frac{q}{Q_{i}} h_{i}\left(\frac{q}{Q_{i}}\right)\left(-\frac{q}{Q_{i}^{2}}\right)\right] \\
- & s_{i}\left[q h_{i}\left(\frac{q}{Q_{i}}\right)\left(-\frac{q}{Q_{i}^{2}}\right)\right. \\
& \left.-Q_{i} \frac{q}{Q_{i}} h_{i}\left(\frac{q}{Q_{i}}\right)\left(-\frac{q}{Q_{i}^{2}}\right)-k_{i}\left(\frac{q}{Q_{i}}\right)\right]-c \\
= & \left(w_{i}+s_{i}\right) k_{i}\left(\frac{q}{Q_{i}}\right)-c .
\end{aligned}
$$




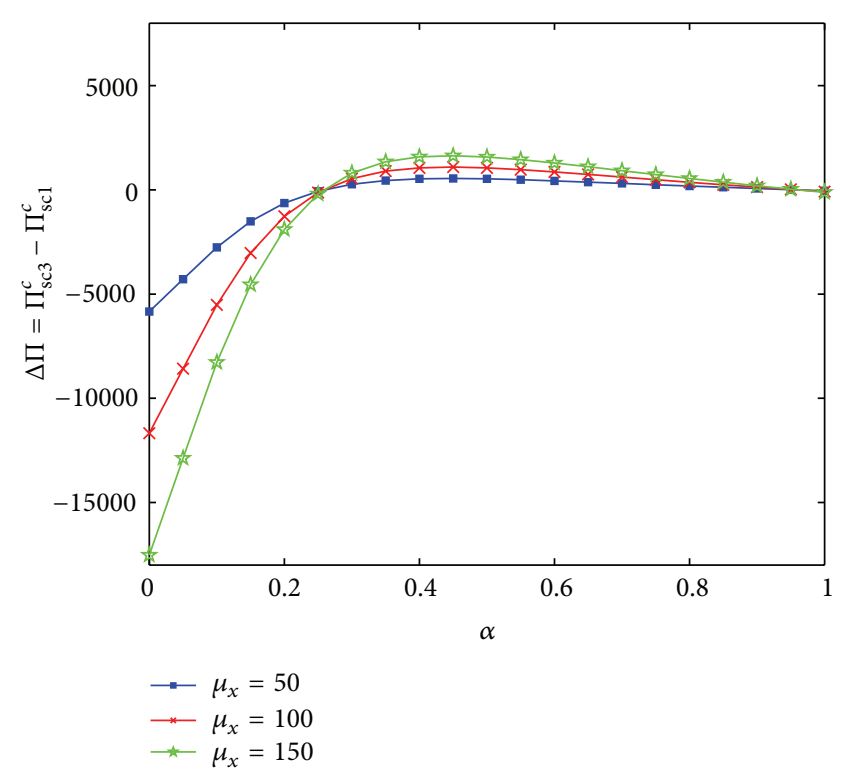

(a)

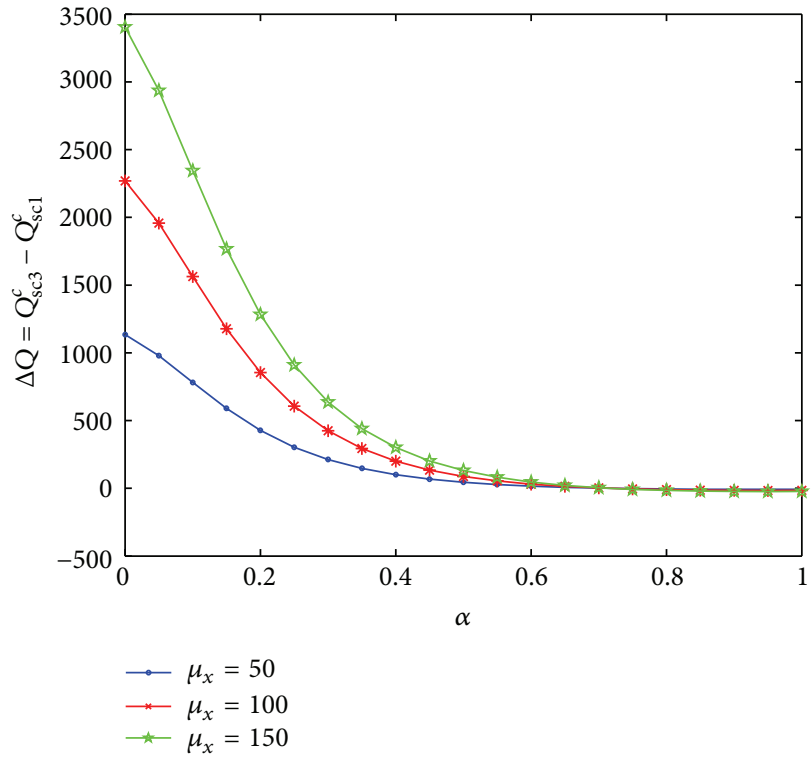

(b)

Figure 5: Variation of $\Delta Q$ and $\Delta \Pi$ with $\alpha$ for different values of $\mu_{x}$.

Developing $\partial \Pi_{s_{i}}^{D} / \partial Q_{i}=0$ leads to the following expression, meanwhile, $\partial^{2} \Pi_{s_{i}}^{D} / \partial Q_{i}^{2}=-\left(w_{i}+s_{i}\right)\left(q^{2} / Q_{i}^{3}\right)<0$ :

$$
\begin{aligned}
& f_{1}\left(Q_{1}, Q_{2}\right)=c-\left(w_{1}+s_{1}\right) k_{1}\left(\frac{q}{Q_{1}}\right), \\
& f_{2}\left(Q_{1}, Q_{2}\right)=c-\left(w_{2}+s_{2}\right) k_{2}\left(\frac{q}{Q_{2}}\right) .
\end{aligned}
$$

For such setting, we always have $\left(\partial f_{1}\left(Q_{1}, Q_{2}\right) / \partial Q_{2}\right)$ $\left(\partial f_{2}\left(Q_{1}, Q_{2}\right) / \partial Q_{2}\right)=-\left(w_{2}+s_{2}\right)\left(q^{2} / Q_{2}^{3} h_{2}\right)\left(q / Q_{2}\right)<0$ for any $Q_{1}$ and $Q_{2}$. Thus, as can be seen from the analysis above, we have the following important observation.

Proposition 2. For any given order quantity from the manufacturer, the sufficient conditions guaranteeing the existence and uniqueness of the Nash equilibrium solution for the suppliers under decentralized scenario are $\left[Q_{1}^{\prime *}, Q_{2}^{\prime *}\right]$.

6.1.2. The Best Response of The Manufacturer. In the next stage, for Stackelberg leader-follower game, we analyze the manufacturer's best response:

$$
\begin{aligned}
\Pi_{m 1}^{D}= & \int_{\underline{\alpha}}^{D / q}\left[\alpha q w-s_{m}(x-\alpha q)\right] h(\alpha) d \alpha \\
& +\int_{D / q}^{\bar{\alpha}} q w h(\alpha) d \alpha-c q,
\end{aligned}
$$

where the first item represents that the manufacturer cannot satisfy the retailer's orders, that is, the manufacturer's sales revenue when $D>\alpha q$. The second item stands for the manufacturer's sales revenue when $D<\alpha q$. From the equation above, we can get $\partial^{2} \Pi_{m 1}^{D} / \partial q^{2}=-\left(w+s_{m}\right)\left(x / q^{3} h\right)(\alpha)<0$, which found that the manufacturer's profit function $\Pi_{m 1}^{D}$ is a concave function about the order quantity $q$ that is, there is only one optimal order quantity $q^{*}$, which makes the manufacturer's profit be the maximum:

$$
\int_{\underline{\alpha}}^{x / q^{*}} \alpha h(\alpha) d \alpha=\frac{c}{w+s_{m}} .
$$

The optimal profit for manufacturer is given by

$$
\Pi_{m 1}^{D *}=w x-\left(w+s_{m}\right) q H\left(\frac{x}{q^{*}}\right) .
$$

6.1.3. The Best Policy of the Retailer. The retailer's expected profit can be written as follow:

$$
\begin{aligned}
\Pi_{r 1}^{D}= & p_{m} \int_{\underline{\beta}}^{\bar{\beta}} E \min (x, \beta q) g(\beta) d \beta \\
& +s \int_{\underline{\beta}}^{\bar{\beta}} E(\beta q-x)^{+} g(\beta) d x d \beta-w q,
\end{aligned}
$$

where $E \min (x, \beta q)=\beta q-\int_{0}^{\beta q}(\beta q-x) f(x) d x$ and $E(\beta q-$ $x)^{+}=\beta q-E \min (x, \beta q)=\int_{0}^{\beta q}(\beta q-x) f(x) d x$; then we simplify above equation:

$$
\Pi_{r 1}^{D}=-\frac{p_{m}-s_{m}}{4 \mu_{x}}\left(\sigma_{\beta}^{2}+\mu_{\beta}^{2}\right) q^{2}+p_{m} \mu_{\beta} q-w q
$$


The optimal ordering quantity and profit of the retailer are, respectively,

$$
\begin{gathered}
q_{1}^{*}=2 \mu_{x} \frac{p_{m} \mu_{\beta}-w}{\left(p_{m}-s\right)\left(\sigma_{\beta}^{2}+\mu_{\beta}^{2}\right)}, \\
\Pi_{r_{1}}^{D *}=\frac{\left(p_{m} \mu_{\beta}-w\right)^{2}}{\left(p_{m}-s\right)\left(\sigma_{\beta}^{2}+\mu_{\beta}^{2}\right)} \mu_{x} .
\end{gathered}
$$

6.2. Optimal Decision in Decentralized Supply Chain with Multi-Agent and RFID Technology. In order to achieve the highest performance of the supply chain, it is necessary to analyze the optimal decision in decentralized supply chain with multi-agent and RFID technology. As we all known, if the retailer invests in information technology, the upstream ones cannot benefit from this, otherwise the opposite. Obviously, from the perspective of the supply chain, it will be better for the whole supply chain with the upstream to make an $\mathrm{R} \& \mathrm{D}$ expenditure. Therefore, here we assume that the technology investment can be actively supported by the suppliers and manufacturer as a whole.

The retailer can benefit from the free-ride RFID adoption and also $\beta=1$. Thus, the retailer's expected profit function is as follow

$$
\begin{aligned}
\Pi_{r 2}^{D}= & p_{m} E \min (x, q)+s_{m} E(q-x)^{+} \\
= & p_{m}\left[q-\int_{0}^{q}(q-x) f(x) d x\right] \\
& +s_{m} \int_{0}^{q}(q-x) f(x) d x-p_{w} q .
\end{aligned}
$$

Since $\partial^{2} \Pi R 2^{D} / \partial q^{2}=-\left(p_{m}-s_{m}\right) f(q)<0$, the profit of retailer is a concave function about $q$. Then, we solve the first derivative and get the corresponding optimal order quantity and total profit of the retailer:

$$
\begin{aligned}
q_{2}^{*} & =2 \mu_{x} \frac{p_{m}-p_{w}}{p_{m}-s_{m}}, \\
\Pi_{r 2}^{D *} & =\frac{\left(p_{m}-p_{w}\right)^{2}}{p_{m}-s_{m}} \mu_{x} .
\end{aligned}
$$

Due to $\sigma_{\beta}>0$ and $0<\mu_{\beta}<1$, it is easy to prove that $\Pi_{r 1}^{D *}<\Pi_{r 2}^{D *}$ is tenable. In another word, after implementing the information technology, the retailer can remotely monitor the inventory and track its position in real time directly, which avoids the impacts of the worse service, inefficient transportation, out of stock or overstock, and other inaccurate inventory problems, so that the retailer needs not undertake cost for this. Thus, the retailer's profit increases after the upstream has deployed the information technology. The Figure 6 respectively shows the impact of changes with $\mu_{x}$ and $c_{t}$ on the variation of $\Pi_{r}^{D}$ and $\Delta \Pi$. We can treat the suppliers and the manufacturer as the same decision-making subject in this section.
Proposition 3. If $c_{t}<c_{t}^{\dagger}$, the manufacturer's profit increases after investing information technology so that he is willing to implement the technology. If $c_{t}>c_{t}^{\dagger}$, the increased cost resulted from the suppliers' application of the multi-agent, and RFID system is larger than the obtained income, so the suppliers and manufacturer have no motivation to invest technology and is unwilling to do, where $c_{t}^{\dagger}=p_{w}-c-\left[p_{w}-\left(p_{w}+\right.\right.$ $\left.\left.s_{m}\right) G\left(q / Q_{1}^{*}\right)\right]\left(p_{m} \mu_{\beta}-p_{w}\right) /\left(\sigma_{\beta}^{2}+\mu_{\beta}^{2}\right)\left(p_{m}-p_{w}\right)$.

It is concluded that $c_{t}^{\dagger}$ is the highest technology cost which can be accepted by the upstream firm in decentralized assembly supply chain. At this time, the income that the technology brings to the suppliers or the manufacturer just equals the newly increased cost resulted from the application of the technology. When the investment cost is higher than $c_{t}^{\dagger}$, the suppliers or manufacturer will not choose to employ the technology actively. On the contrary, if this cost is lower than $c_{t}^{\dagger}$, the income that the supplier or manufacturer obtains by information technology is higher than the technology investment, so that they will all have enormous enthusiasm in investing it.

According to the above propositions, in the situation of $c_{t}^{\prime}>c_{t}>c_{t}^{\dagger}$, although the suppliers' application of information technology can increase the profit of the supply chain, his profit will reduce. Furthermore, the upstream firm has no motivation to use multi-agent and RFID system so that the whole supply chain cannot profit from the technology and the maximum profit of the supply chain cannot be realized. Thus, it is necessary to study the supply chain coordination in the situation of $c_{t}^{\prime}<c_{t}<c_{t}^{\dagger}$ to make the supplier have enthusiasm to adopt this technology and reduce the inaccuracy of the supply chain inventory as well as increase the performance of the supply chain and its members.

\section{Numerical Analysis}

Finally, a numerical example is taken to validate the rationality and feasibility of the theoretics and method. When the customer demand follows uniform distribution pattern, $x \sim U(0,200)$, we insert the following constant values into the corresponding optimal expressions: $p_{w}=20, c=12$, $p_{w}=20, p_{m}=30, v=8$, and $s_{m}=5$, when $0<c_{t}<7.8$, the upstream ones are unwilling to use RFID and multi-agent technology because the profit will be reduced after adopting it.

Furthermore, according to Figure 7, we can find that the increased profit of the manufacturer and the retailer is a linear function about $\beta$. The increased profit of the manufacturer monotonously reduces as the shelf inventory error rate increases, while the increased profit of the retailer monotonously increases as the coefficient $\beta$ increases. When the factor of the inventory error $0<\beta<0.4$, the manufacturer's profit is beneficial from reasonable utilization of information technology; at this time, the upstream firm is willing to make a investment. If $\beta>0.2$, the retailer's profit increases after he has deployed RFID and multiagent technology. Therefore, choosing the factor of the shelf inventory error rate at the interval of $0.2<\beta<0.4$ can 


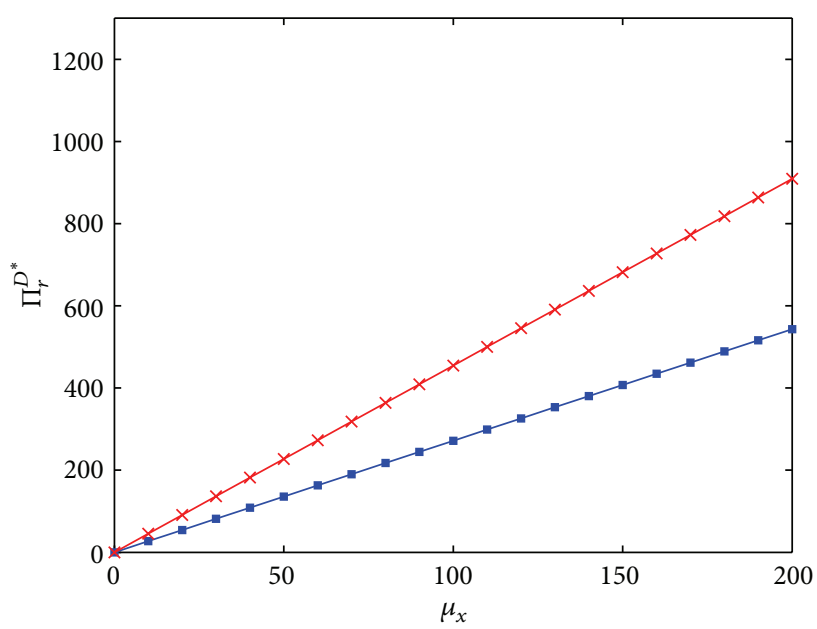

$$
\begin{aligned}
& -\Pi_{r_{1}}^{D^{*}} \\
& -\Pi_{r_{2}}^{D^{*}}
\end{aligned}
$$

(a)

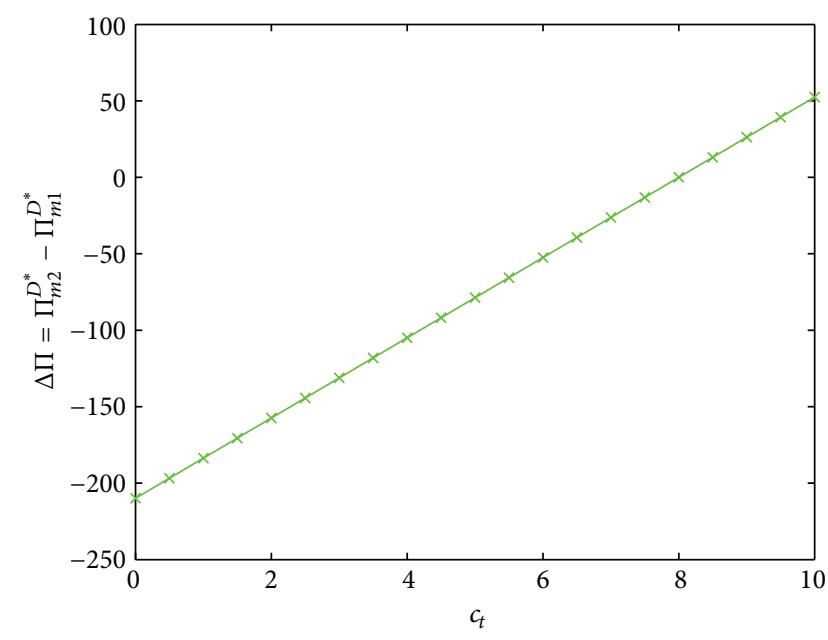

(b)

FIgURE 6: Variation of $\Pi_{r}^{D}$ with $\mu_{x}$ and $\Delta \Pi$ with $c_{t}$.

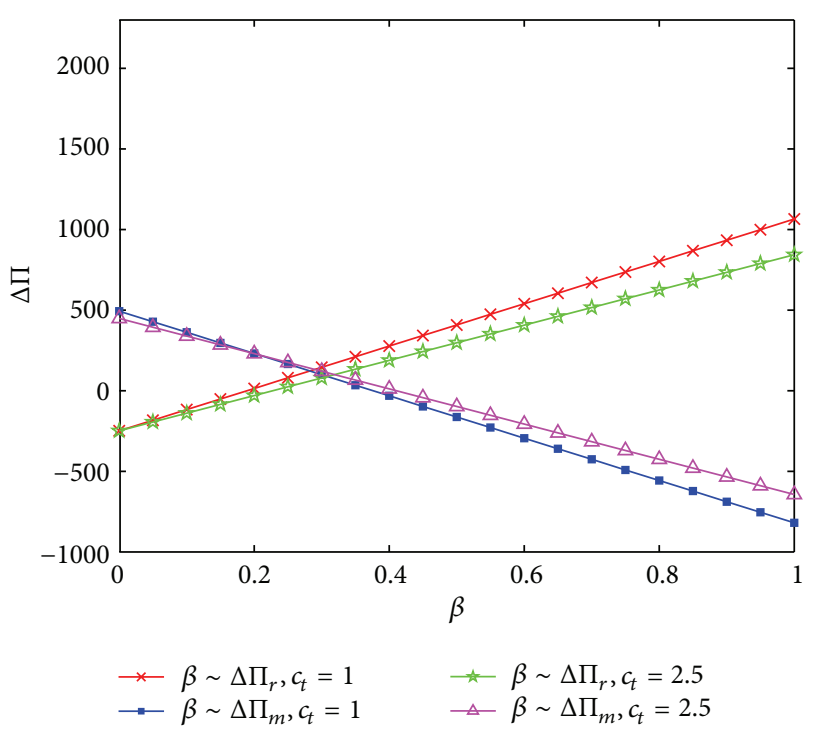

FIGURE 7: Variation of $\Delta \Pi_{r}$ and $\Delta \Pi_{m}$ with $\beta$ for different values of $c_{t}$.

ensure that the profit increases to make both the upstream and downstream firm achieve a win-win situation.

Moreover, the suppliers aim at encouraging the retailer to order more goods, but the retailer directs at freely riding the innovative technology from the upstream ones. Thus, they can consider the coordination model based on the revenue sharing. That is, the suppliers and manufacturer undertake the technology investment cost alone and give price subsidy to the retailer on the condition that the wholesale price is not changed. Or the retailer gives parts of sales revenue to the upstream ones at the end of a sales season in order to stimulate and encourage the suppliers to increase the R\&D investment.

Being restrained by personal reason, the members of the supply chain will first consider their own benefit and will take the maximization of the benefit of the whole supply chain into consideration on the premise that their own benefit is satisfied. Thus, to ensure that the members are willing to accept the revenue sharing contract, it is necessary to guarantee that the profit obtained by both parties under this contract with the supply chain that has adopted this technology is not less than the one that has been obtained in decentralized scenario before the contract is made. In other words, it is worth considering to ensure that the members of the supply chain can achieve win-win situation with the restraint of the contract. In other words, the coordination mechanism is to ensure the following two inequalities tenable: $\Delta \Pi_{r}>0$ and $\Delta \Pi_{m}>0$.

\section{Concluding Remarks}

The information technology has been heralded as a major breakthrough to enhance the efficiency of assembly supply chain. This paper mainly aims to explore the impact of information technology on supply chain management, especially the prospects of multi-agent and RFID investment value. Meanwhile, the possibility and reliability of the model are examined through the qualitative and quantitative analyse of the multi-agent and RFID system investment. Additionally, supply chain partnership is an important factor of performance in supply chain operations. It is significant to strengthen the supply chain cooperation management. Finally, this paper mainly aims at a perfectly competitive market and carries out the research in the situation that the market demands are uniformly distributed. Future researches 
should focus on whether the revenue sharing contract can coordinate the supply chain when the market demands are normal distribution. As is apparent from the above description, the research of this paper has important theoretical value and great practical significance.

\section{Notations}

$\begin{array}{ll}x: & \text { The random variable representing the } \\ & \text { customer's demand } D \\ p_{m}: & \text { The manufacturer's selling price per item } \\ q_{m}: & \text { The ordering quantity of manufacturer } \\ s_{m}: & \text { The unit production shortage cost of } \\ & \text { manufacturer } \\ v: & \text { The salvage cost per item } \\ Q_{i}: & \text { The supplier's production quantity } i=1,2 \\ c: & \text { The unit production cost of supplier } i \\ w_{i}: & \text { The wholesale price of supplier } i \\ \alpha: & \text { The errors in assembly system (process) } \\ h(H): & \text { Pdf(cdf) characterizing } \alpha \\ \beta: & \text { The shelf inventory error rate (caused by } \\ g(G): & \text { Pdf(cdf) characterizing } \beta \\ c_{t}: & \text { The technology investment cost (involving } \\ & \text { multi-agent software and RFID tag cost) } \\ \Pi_{\mathrm{sc}}^{c}: & \text { The expected profit of whole supply chain } \\ & \text { in centralized scenario }\end{array}$

\section{Acknowledgments}

This research was supported in part by the National Natural Science Foundation of China under Grant no. 51175187, the National High-Tech. R\&D Program of China under Grant no. 2007AA04Z111, and the Grant of the University of Macau MYRG163 (Y1-L3) FBA11-LZT.

\section{References}

[1] S. Tzeng, W. Chen, and F. Pai, "Evaluating the business value of RFID: evidence from five case studies," International Journal of Production Economics, vol. 112, no. 2, pp. 601-613, 2008.

[2] A. Ustundag and M. Tanyas, "The impacts of radio frequency identification (RFID) technology on supply chain costs," Transportation Research E, vol. 45, no. 1, pp. 29-38, 2009.

[3] U. Bagchi, A. Guiffrida, L. O’Neill, A. Zeng, and J. Hayya, “The effect of RFID on inventory management and control," in Trends in Supply Chain Design and Management, pp. 71-92, 2007.

[4] H. Lee and Ö. Özer, "Unlocking the value of RFID," Production and Operations Management, vol. 16, no. 1, pp. 40-64, 2007.

[5] A. Atali, H. Lee, and Ö. Özer, "If the inventory manager knew: value of visibility and RFID under imperfect inventory information," Social Science Research Network, 2009.

[6] M. Tajima, "Strategic value of RFID in supply chain management," Journal of Purchasing and Supply Management, vol. 13, no. 4, pp. 261-273, 2007.
[7] E. W. T. Ngai, K. K. L. Moon, F. J. Riggins, and C. Y. Yi, "RFID research: an academic literature review (1995-2005) and future research directions," International Journal of Production Economics, vol. 112, no. 2, pp. 510-520, 2008.

[8] M. M. Hossain and V. R. Prybutok, "Consumer acceptance of RFID technology: an exploratory study," IEEE Transactions on Engineering Management, vol. 55, no. 2, pp. 316-328, 2008.

[9] E. W. T. Ngai, T. C. E. Cheng, S. Au, and K. Lai, "Mobile commerce integrated with RFID technology in a container depot," Decision Support Systems, vol. 43, no. 1, pp. 62-76, 2007.

[10] G. M. Gaukler, R. W. Seifert, and W. H. Hausman, "Item-level RFID in the retail supply chain," Production and Operations Management, vol. 16, no. 1, pp. 65-76, 2007.

[11] H. S. Heese, "Inventory record inaccuracy, double marginalization, and RFID adoption," Production and Operations Management, vol. 16, no. 5, pp. 542-553, 2007.

[12] A. Dutta, H. L. Lee, and S. Whang, "RFID and operations management: technology, value, and incentives," Production and Operations Management, vol. 16, no. 5, pp. 646-655, 2007.

[13] Y. Rekik, E. Sahin, and Y. Dallery, "Analysis of the impact of the RFID technology on reducing product misplacement errors at retail stores," International Journal of Production Economics, vol. 112, no. 1, pp. 264-278, 2008.

[14] J. G. Szmerekovsky and J. Zhang, "Coordination and adoption of item-level RFID with vendor managed inventory," International Journal of Production Economics, vol. 114, no. 1, pp. 388398, 2008.

[15] E. Bottani and A. Rizzi, "Economical assessment of the impact of RFID technology and EPC system on the fast-moving consumer goods supply chain," International Journal of Production Economics, vol. 112, no. 2, pp. 548-569, 2008.

[16] Y. Tu, W. Zhou, and S. Piramuthu, "Identifying RFID-embedded objects in pervasive healthcare applications," Decision Support Systems, vol. 46, no. 2, pp. 586-593, 2009.

[17] S. Wang, S. Liu, and W. Wang, "The simulated impact of RFIDenabled supply chain on pull-based inventory replenishment in TFT-LCD industry," International Journal of Production Economics, vol. 112, no. 2, pp. 570-586, 2008.

[18] J. Whitaker, S. Mithas, and M. S. Krishnan, "A field study of RFID deployment and return expectations," Production and Operations Management, vol. 16, no. 5, pp. 599-612, 2007. 


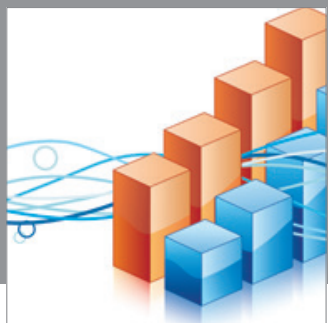

Advances in

Operations Research

mansans

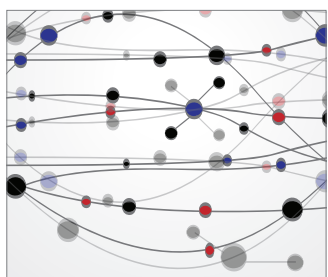

The Scientific World Journal
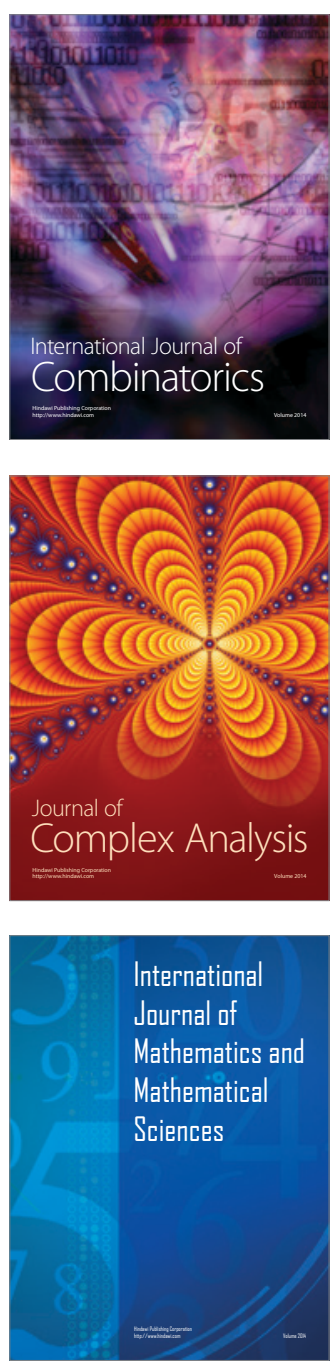
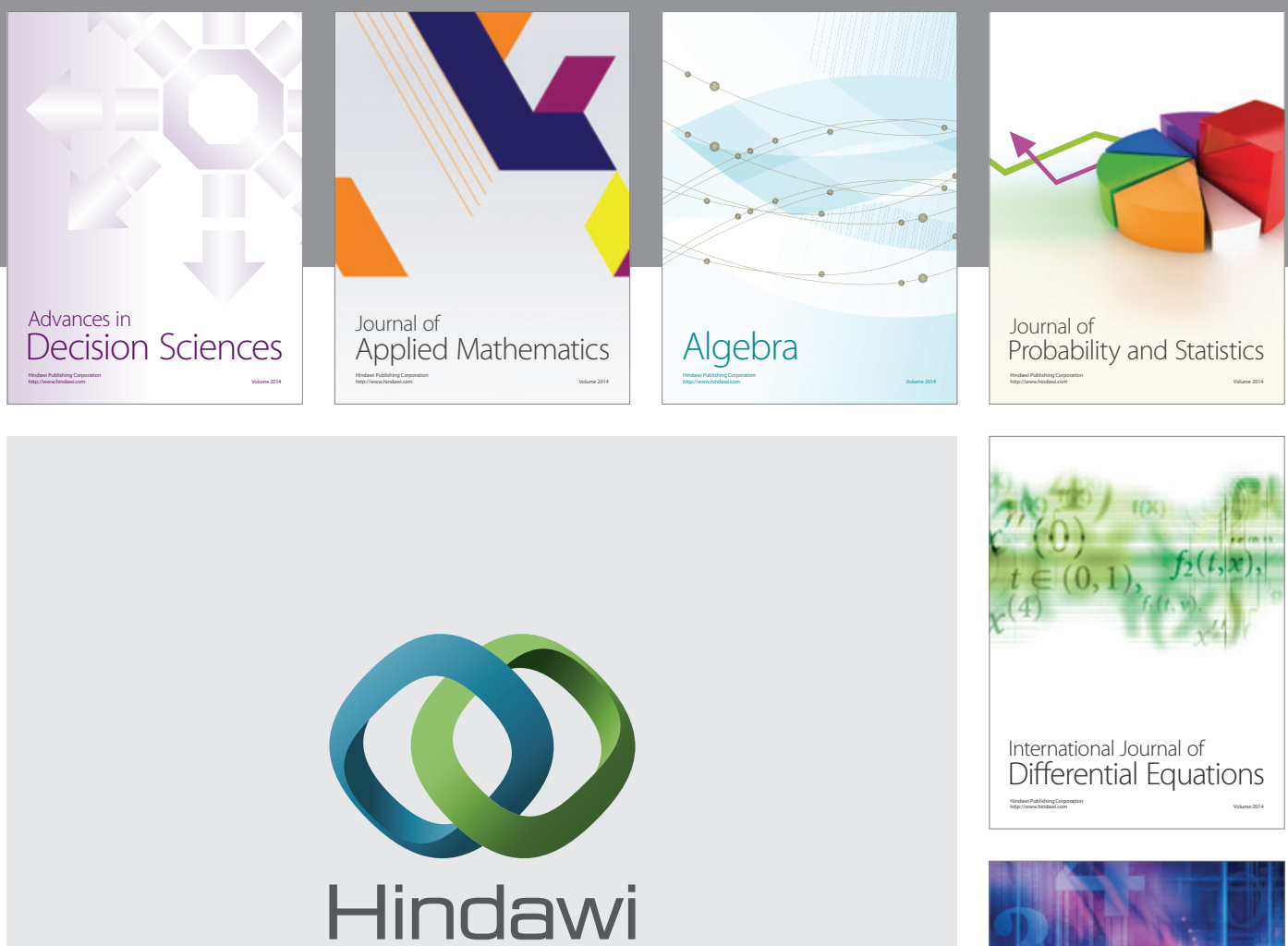

Submit your manuscripts at http://www.hindawi.com
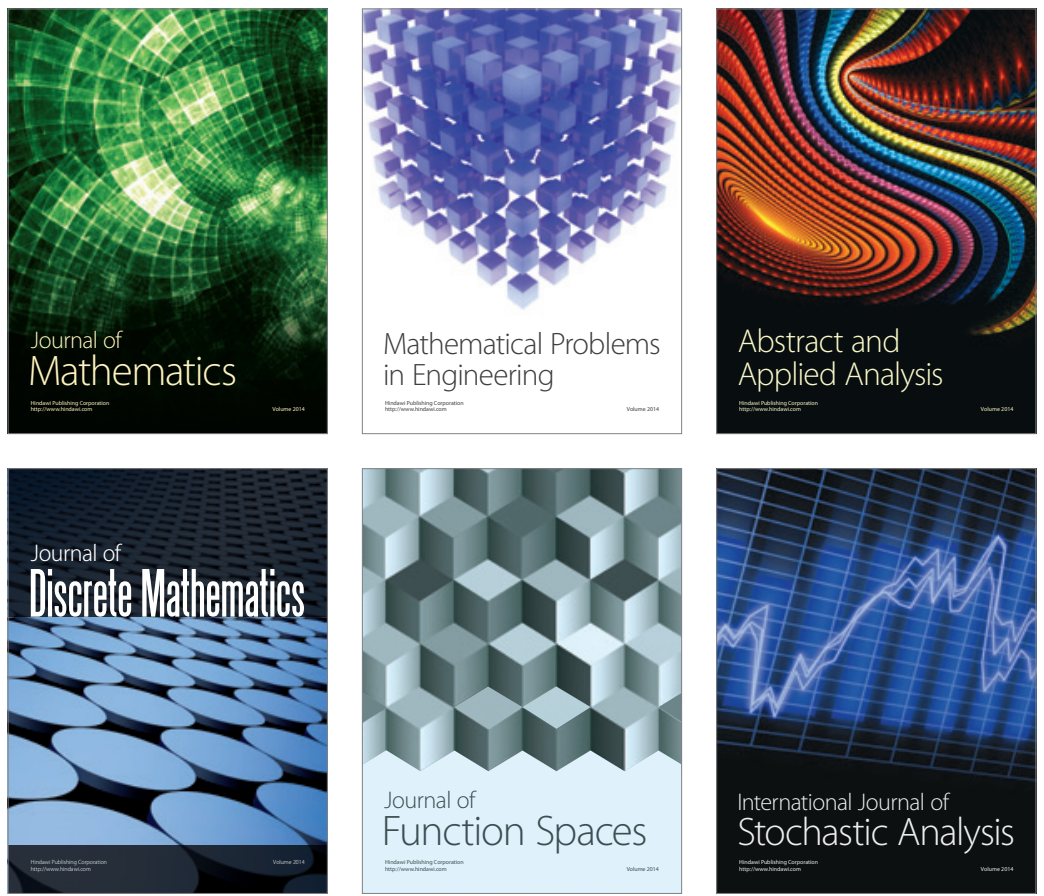

Journal of

Function Spaces

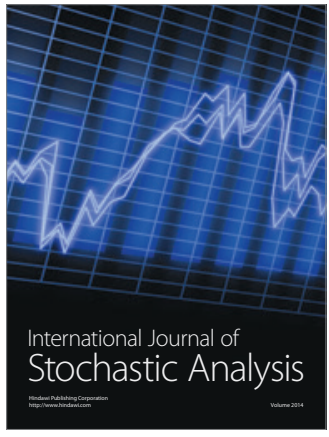

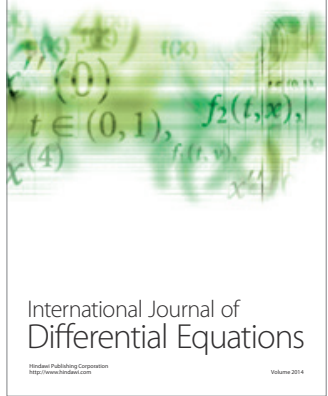
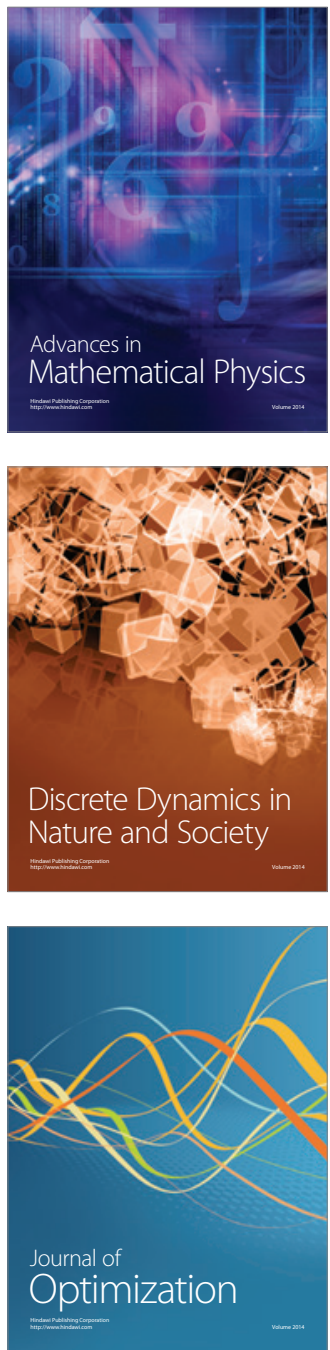